\title{
Impact of maternal depressive symptoms on the development of infant temperament: Cascading effects during the first year of life
}

\author{
Silvia Rigato $^{1}$ (D) | Manuela Stets ${ }^{2}$ (D) | Arielle Bonneville-Roussy ${ }^{3}$ (D) | \\ Karla Holmboe ${ }^{4}$ (D)
}

${ }^{1}$ Department of Psychology, Centre for Brain Science, University of Essex, Colchester, UK

${ }^{2}$ Department of Psychology, Anglia Ruskin University, Cambridge, UK

${ }^{3}$ School of Education, University of Roehampton, London, UK

${ }^{4}$ Department of Experimental Psychology, University of Oxford, Oxford, UK

\section{Correspondence}

Silvia Rigato, Department of Psychology, Centre for Brain Science, University of Essex, Wivenhoe Park, Colchester, CO4 3SQ, UK.

Email: srigato@essex.ac.uk

Funding information

Bill and Melinda Gates Foundation,

Grant/Award Number: Grand Challenges Explorations (OPP1119448)

\begin{abstract}
Maternal depression is associated with a range of child development outcomes, including difficult temperament. This longitudinal study investigated whether depressive symptoms (DS) that mothers experience after childbirth predict infant negative affect (NA), as well as potential effects of infant NA on maternal DS, across the first year of life. In the study $(N=63$ ), questionnaires (the Beck Depression Inventory Second Edition, and the Infant Behavior QuestionnaireRevised Very Short Form) were administered at 2 weeks, 4, 6 , and 9 months after birth. Using path analysis, we tested five different models that could explain the relationship between maternal DS and infant NA. The best-fitting model showed that the days immediately following childbirth represent an important time for the development of infant temperament as maternal mood significantly predicts infant NA for at least 4 months after birth. This does not constitute a single sensitive period; a new predictive effect emerges around 4 months of age, suggesting cascading influences of maternal DS across the first 6 months of life. These results suggest a need for support, should a mother experience DS, not only immediately after birth, but also throughout the early stages of parenting.
\end{abstract}




\section{KEYWORDS}

individual differences, infant temperament, longitudinal, maternal depression, negative affect, structural equation modeling (SEM)

\section{1 | INTRODUCTION}

Between 11 and 29\% of women experience depression and/or stress during pregnancy or after giving birth (Howard et al., 2014; Priest, Austin, Barnett, \& Buist, 2008; Weisman et al., 2010). Furthermore, a sizeable proportion of new mothers experience subclinical postnatal depressive symptoms (DS) (e.g., 20\% in Cutrona \& Troutman, 1986; 11\% in Cents et al., 2013; 40\% in Giallo, Woolhouse, Gartland, Hiscock, \& Brown, 2015; 44\% in Shapiro, Jolley, Hildebrandt, \& Spieker, 2018). Importantly, a substantial proportion of women continue to have DS into their child's early years (e.g., Horwitz, Briggs-Gowan, Storfer-Isser, \& Carter, 2009; Woolhouse, Gartland, Mensah, \& Brown, 2015).

Prenatal and postnatal maternal mental health difficulties, such as maternal depression, are associated with long-term negative effects on the mother, as well as increased risk of suboptimal developmental outcomes during the neonatal period, infancy and childhood, from insecure attachment to difficult temperament, behavioral problems, and cognitive deficits (e.g., Beck, 1996, 1998; Kingston, McDonald, Austin, \& Tough, 2015; Kingston \& Tough, 2014; Kingston, Tough, \& Whitfield, 2012; Murray, Halligan, \& Cooper, 2010; Pawluski, Lonstein, \& Fleming, 2017). The association between maternal depression and problematic offspring outcomes are often found to last well into adolescence and adulthood (Campbell, Morgan-Lopez, Cox, McLoyd, \& NICHD-ECRN, 2009; Weissman et al., 2016). Given these long-lasting associations, it is surprising that only a few studies have focused on the very earliest potential influence of maternal mental health (e.g., Granat, Gadassi, Gilboa-Schechtman, \& Feldman, 2017; Shapiro et al., 2018). This is particularly striking, given evidence suggesting that, even when symptom severity is low, exposure to maternal DS predicts child behavioral and emotional problems (Cents et al., 2013; Giallo et al., 2015; Shapiro et al., 2018). In the current longitudinal study, we aimed to shed further light on the relationship between maternal DS and infant negative affect (NA) in a nonclinical general population sample. Measures were taken longitudinally from 2 weeks to 9 months postpartum.

Theorists within the field have tried to characterize the potential pathways from maternal depression to negative outcomes for the child. For example, Goodman and Gotlib (1999) developed a conceptual model whereby four primary mechanisms (heritability of depression, innate dysfunctional neuroregulatory mechanisms, exposure to negative maternal cognition, behaviors and affect, and exposure to stressful events) are more likely to be present when a mother is depressed and can lead to the emergence of cognitive and affective vulnerabilities in the offspring. The interactions of such vulnerabilities with various internal (e.g., child temperament) and external (e.g., father's availability and mental health) moderators determine the likelihood of the child developing psychopathology later in life.

In terms of infant temperament more specifically, previous research has found that infants of depressed mothers show more NA (e.g., Feldman et al., 2009; McMahon, Barnett, Kowalenko, Tennant, \& Don, 2001; Whiffen \& Gotlib, 1989). NA refers to the tendency to experience and express the emotions of frustration/anger, fear, and sadness (Goldsmith et al., 1987). High NA therefore characterizes those infants who are inclined to cry or fuss, show more negative emotions overall, and who exhibit intense emotional reactions (Rothbart \& Bates, 2006). However, because depressed mothers also express more NA and show less positive engagement and responsiveness (Campbell, Cohn, \& Meyers, 1995; Field, 1984; Lyons-Ruth, Connell, Grunebaum, \& Botein, 1990; Pickens \& Field, 1993), this potentially leads to a negative self-reinforcing interaction pattern (Cummings \& Davies, 1994; Tronick, 1989). In fact, detailed observational research investigating the microlevel interactions between depressed mothers and their infants has shown differences between depressed mothers and controls in terms of less sensitive responding, shorter gaze durations toward the infant, slower gaze-touch synchrony, and atypical 
coordination of gaze synchrony. Similarly, infants of depressed mothers (compared to controls) display less social engagement, more gaze aversion, atypical gaze synchrony, and less emotional buffering from maternal presence (Beebe et al., 2008; Feldman et al., 2009; Granat et al., 2017).

These findings clearly suggest a link between maternal DS and infant NA, and also point to some of the potential mechanisms underlying this association. However, most previous research has been cross-sectional in nature (e.g., Granat et al., 2017), and limited research exists on the development of the association between maternal DS and infant NA during early infancy (but see, Shapiro et al., 2018). An open question that remains is whether the driving factor in the association between maternal DS and infant NA comes from the mother or the infant. It may be that infants of depressed mothers primarily experience and express more NA as a result of the mother's depressed mood, but it could also be the case that temperamentally "difficult" babies elicit feelings of depression and inadequacy in the mother. A third possibility is that both effects are present and interact with each other. The literature in this area so far has provided evidence for all three scenarios, including contrasting patterns of association.

In terms of infant-driven effects, Cutrona and Troutman (1986) presented evidence from a general population sample for a model whereby difficult infant temperament, characterized by intense crying, lack of soothability, and unpredictability, increased the risk of depression in the mother 3 months postpartum. Similarly, Murray, Stanley, Hooper, King, and Fiori-Cowley (1996) found that mothers who were at high risk of depression were particularly likely to develop postnatal depression if their infant was highly irritable during the first few days after birth. Specifically, high irritability (as well as poor motor scores) assessed in the neonatal period was a strong predictor of the onset of maternal depression 8 weeks after childbirth (Murray et al., 1996). The mechanisms behind such infant-driven effects are yet to be fully established, but Cutrona and Troutman (1986) found that mothers who reported a high level of social support also experienced fewer DS and a higher level of self-confidence in their parenting role. This suggests that social support may impact on maternal mood and play a mediating protective role against depression through its influence on parenting self-efficacy. It is also worth mentioning that the mother's perception of her infant's level of NA rather than objectively measured infant NA, could be a driving factor in creating a sense of low parenting self-efficacy and depressed mood in the mother.

Other research has revealed the opposite pattern of association, with maternal DS predicting infant NA. In a Japanese study (Sugawara, Kitamura, Toda, \& Shima, 1999), maternal postnatal depression was self-assessed at 5 days and 12 months postpartum, and infant temperament was assessed at 6 and 18 months. Among five temperamental dimensions, only those relating to negative affectivity were found to be associated with maternal depression; specifically, maternal depression 5 days after childbirth predicted lower infant frustration tolerance at 6 months. Similarly, in a more recent study, Shapiro and colleagues (2018) found evidence that postpartum depression at 3 weeks predicted infant NA at 6 months.

Research into the potential mechanisms behind maternally-driven effects (i.e., effects of maternal DS on infant NA) has suggested that the quality of parent-infant interaction plays an important role. For instance, whereas high parental sensitivity, that is, the ability to promptly respond to and correctly interpret the child's cues (Ainsworth, Blehar, Waters, \& Wall, 1978), facilitates the development of the child's ability to control NA (Kopp, 1989), a more intrusive parenting style may lead to an increased level of NA (Belsky, Putnam, \& Crnic, 1996). Further, mothers experiencing DS develop altered and potentially problematic patterns of interaction with their infant, resulting in a suboptimal parenting style (e.g., Cummings \& Davies, 1994; Field, Healy, Goldstein, \& Guthertz, 1990; Gordon et al., 1989; Tronick, 1989).

Finally, there is also evidence for more complex effects. As mentioned previously, researchers investigating maternal depression in relation to child outcomes have long hypothesized that adverse bi-directional or transactional influences are likely to occur between mother and infant when the mother is depressed and the infant has a difficult temperament; that is, where one communication partner reinforces the negative response of the other (e.g., Tronick, 1989), potentially leading to a vicious circle (Cummings \& Davies, 1994; Murray, Halligan, \& Cooper, 2010). Investigating the longitudinal associations between child temperament and maternal stress (a construct similar to maternal DS), Pesonen et al. (2008) found evidence for bi-directional effects. However, the effect of 
maternal stress on child temperament was greater; specifically, maternal stress when infants were 6 months old predicted an increase in child NA between infancy and 5 years of age. A more recent study involving direct observation of 388 mother-infant dyads similarly suggested a transactional predictive relationship in that infant NA at 5 months predicted maternal intrusiveness at 10 months, which in turn predicted increased toddler NA at 24 months (Perry, Dollar, Calkins, \& Bell, 2018).

It should be clear from this review, that there is evidence for maternally-driven, infant-driven and bi-directional/transactional effects in the relationship between maternal DS and infant temperament. Given the consistent association between maternal DS and long-term child outcomes (see e.g., Kingston et al., 2015), it is of great importance to identify the underlying mechanisms as they unfold over developmental time. Yet, at present, knowledge is limited regarding the very earliest interactions between maternal DS and infants' development and expression of their own negative emotions. This is partly due to the lack of studies investigating the effect of maternal depression longitudinally, that is, not just at birth or at a single time point during infancy, but repeatedly across the first year to establish the timing of effects. The need for meticulous longitudinal studies with a dense measurement strategy has been highlighted in previous research, in particular, the importance of repeated assessments starting in the early postpartum period (e.g., Beck, 1996; Brennan et al., 2000; Shapiro et al., 2018; Sugawara et al., 1999). Furthermore, understanding more about the timing and directionality of effects will be useful in developing targeted interventions. The current study, by tracking maternal DS in relation to infant temperament across the first year of life, may help identify the crucial time (or times) when maternal DS start to predict the development of infant NA or vice versa.

\section{1 | The current study}

In an effort to elucidate the important interactions between maternal depression level and infant NA over time, we employed a longitudinal design and used identical measures and the same mode of data collection at four age points after birth. We focused on mothers' DS in a general population sample (measured by the Beck Depression Inventory Second Edition; Beck, Steer, \& Brown, 1996) and on infant NA across the first year of life (measured by the Infant Behavior Questionnaire-Revised Very Short Form; Putnam, Helbig, Gartstein, Rothbart, \& Leerkes, 2014). Maternal DS and infant temperament were both assessed via online questionnaires sent to the mother 2 weeks after birth, as well as when the infant was 4 months, 6 months and 9 months of age. At 9 months, we additionally asked mothers to complete a questionnaire on (a) their parental feelings and (b) parental attitudes (brief instruments based on scales developed by Deater-Deckard, 2000; and Budd, Danko, Li, \& Henriquez, 2015), in order to broadly assess the potential association between parenting factors, maternal DS and infant NA.

In our main analyses, we used path analysis within a structural equation modeling (SEM) framework (Kline, 2016) to test five models evaluating the direction of the associations between maternal DS and infant NA across the first year of life. In line with previous evidence that shows predictive effects of maternal DS on infant NA (e.g., Shapiro et al., 2018; Sugawara et al., 1999), we tested two maternally-driven models. In Model 1, maternal DS around the time of birth had a primary and exclusive effect on later infant NA (Sensitive Period Effects of Maternal DS, Figure 1). In Model 2, effects were set to be cascading, that is, new effects of maternal DS emerged a few months after a change in maternal mood (Delayed Effects of Maternal DS, Figure 2). Based on evidence pointing to the reverse pattern of association in the early period following childbirth (Cutrona \& Troutman, 1986; Murray et al., 1996), we also tested an alternative infant-driven model. In this model (Model 3), infant NA had cascading effects on maternal DS (Delayed Effects of Infant NA, Figure 3). Based on the substantial evidence for predictive links between maternal DS and infant NA, we expected that Models 1-3 would fit the data better than a control model involving stability in each measure and within-age associations between maternal DS and infant NA, but without there being any longitudinal associations between the two variables (Model 4: Stability Model, Figure 4). Finally, based on the evidence showing bi-directional and transactional influences between mother and 
Model 1

Sensitive Period

Effects of Maternal

Depressive

Symptoms

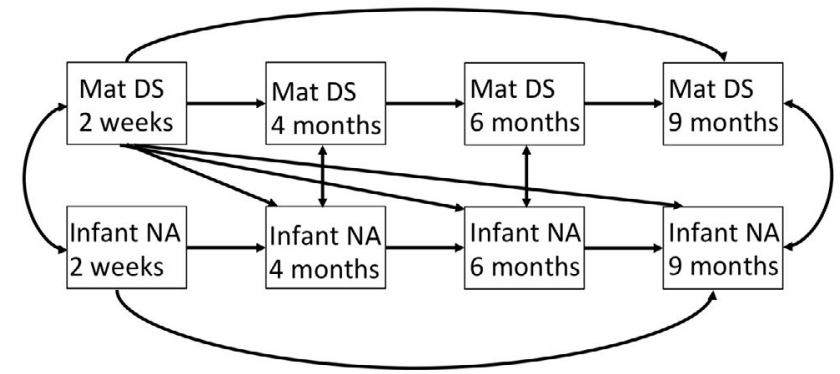

FIGURE 1 Representation of Model 1: Sensitive Period Effects of Maternal Depressive Symptoms: Maternal depressive symptoms (DS) at 2 weeks after childbirth predict infant negative affect (NA) at all ages assessed

Model 2

Delayed Effects

of Maternal

Depressive

Symptoms

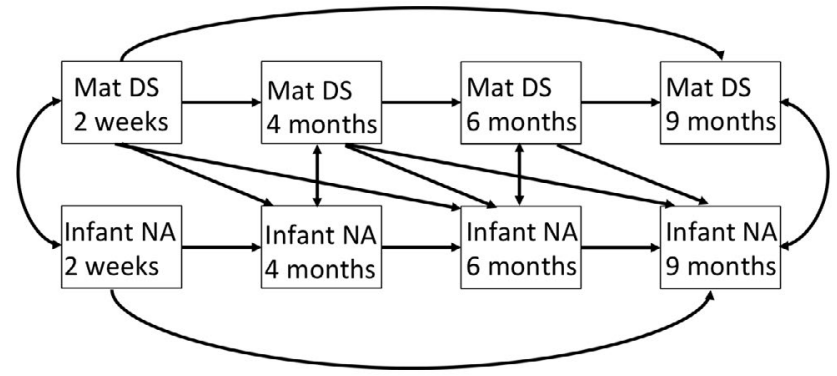

FIGURE 2 Representation of Model 2: Delayed Effects of Maternal Depressive Symptoms: (1) Maternal depressive symptoms (DS) at 2 weeks predict infant negative affect (NA) at 4 and 6 months, (2) maternal DS at 4 months predict infant NA at 6 and 9 months, and (3) maternal DS at 6 months predict infant NA at 9 months

Model 3 Delayed Effects of Infant Negative Affect

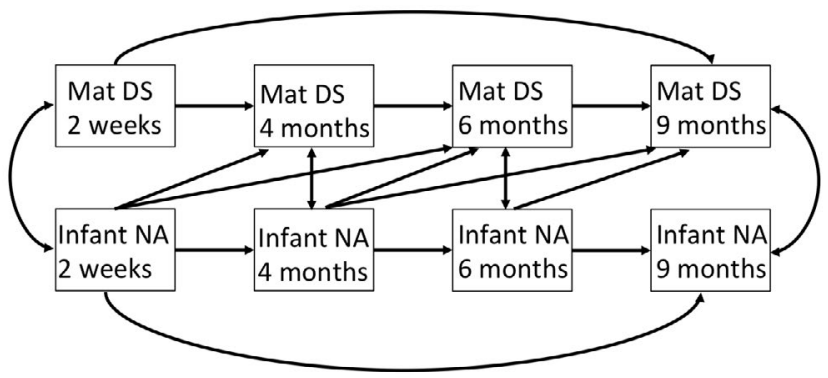

FIGURE 3 Representation of Model 3: Delayed Effects of Infant Negative Affect: (1) Infant negative affect (NA) at 2 weeks predicts maternal depressive symptoms (DS) at 4 and 6 months, (2) infant NA at 4 months predicts maternal DS at 6 and 9 months, and (3) infant NA at 6 months predicts maternal DS at 9 months

infant (Perry et al., 2018; Pesonen et al., 2008), we tested a more complex bi-directional model with cascading longitudinal paths between maternal DS and infant NA (Model 5: Bi-directional Model, Figure 5). Essentially, this model was a combination of Models 2 and 3. Given the additional paths included in this model, and the need for a larger sample size when more complex models are tested, the latter model should be considered more exploratory than the other models and in need of further testing in the future. All models included within-age correlations between maternal DS and infant NA, as well as a longitudinal within-measure path for each measure (maternal DS and infant NA) between 2 weeks and 9 months. The latter paths were included due to high stability in both measures across the assessment period. 
Model 4

Stability Model

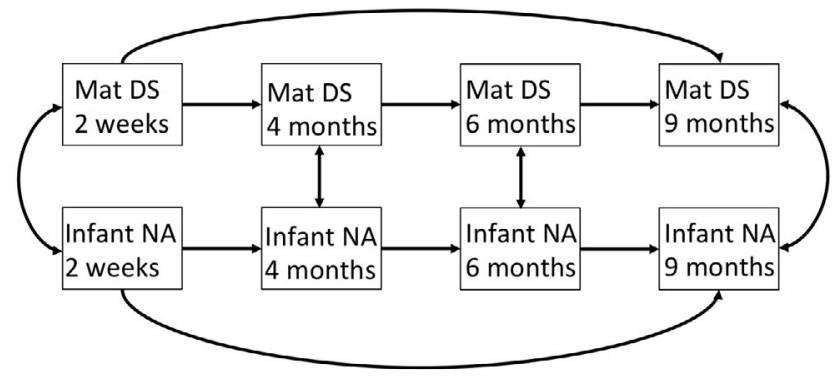

FIGURE 4 Representation of Model 4: Stability of Maternal Depressive Symptoms and Infant Negative Affect (control model): Maternal depressive symptoms (DS) and infant negative affect (NA) are stable over time and correlate within each age, but there are no predictive associations between the two measures

Model 5

Bi-directional

Model

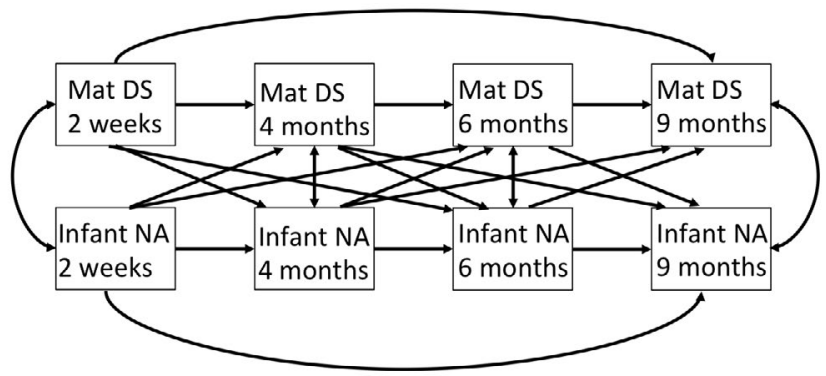

FIGURE 5 Representation of Model 5: Delayed Effects of both Maternal Depressive Symptoms and Infant Negative Affect (Bi-directional Model): Models 2 and 3 combined, i.e., maternal depressive symptoms (DS) predict later infant negative affect (NA) and infant NA predicts later maternal DS

\section{2 | METHODS}

\section{1 | Participants}

A total of 81 families were recruited from the University of Essex Babylab database and through events for expectant parents at Colchester General Hospital and other local venues in Essex, U.K. Ethical approval was gained from the National Health Service's National Research Ethics Service Committee, London-Hampstead branch, U.K. (Ref. No. 15/LO/0478). The approved protocol included a procedure for guiding mothers at high risk of clinical depression to further support and treatment options.

The current study reports on questionnaire data from a larger longitudinal study investigating attention and social skills in the first year of life using multiple methods (behavioral, neuroimaging and questionnaires), and consisting of one pre- and four postnatal assessment points. The postnatal lab sessions were scheduled at the following ages: 2 weeks, 4, 6, and 9 months after birth. Of the 81 recruited families, 16 mothers participated only in the prenatal assessment, and could therefore not be included in the current analyses. Additionally, two infants suffered from complications at birth, and were excluded from the final sample. The sample reported on here therefore consisted of 63 infants and their mothers. The lab-based longitudinal data from these infants is the topic of separate reports.

The majority of participating infants were born full-term (at 37 weeks of gestation or later; $M=40$ weeks, 3 days), and only two infants were born between 36 and 37 weeks of gestation. Infants in the sample had normal birth weight $(M(S D)=3.5(0.4) \mathrm{kg}$; Range $=2.44-4.82 \mathrm{~kg})$, no complications at birth, and no known health issues (pregnancy, birth and health information was missing for four infants). At the time of the prenatal assessment, 
which occurred around 36 weeks of gestation, mothers were on average 31.2 years of age $(S D=4.6)$; fathers were on average 33.6 years of age $(S D=6.1)$. Mothers had spent an average of 16.8 years in education $(S D=3.6)$ and fathers had spent an average of 15.2 years in education $(S D=3.7)$. The infants' ethnic backgrounds were mainly White British ( $N=54 ; 85.7 \%$ ). Three infants were of Other White background (4.8\%), four infants had mixed ethnic backgrounds but did not provide further details (6.4\%), one infant was White British mixed with another ethnic background (1.6\%), and one infant had a Hispanic background (1.6\%).

\section{2 | Questionnaires}

Participating mothers were asked to complete well-established questionnaires relating to infant temperament as well as symptoms of depression. A questionnaire on parenting feelings and attitudes was also included in the protocol. Details on each of the questionnaires are provided below. The links to the online questionnaires were sent via email to those families who had confirmed attendance for a scheduled lab test session for the following day in an effort to keep the time difference between test session and questionnaire completion as short as practically possible. Details on infant age at the time of questionnaire completion and questionnaire completion rates are listed in Table 1. Anonymized individual scale scores for the Infant Behavior Questionnaire-Revised Very Short Form and the Beck Depression Inventory Second Edition can be found in the Supporting Information in Table S1. These data are also available on https://osf.io/mg68h/.

\subsection{1 | The Infant Behavior Questionnaire-Revised Very Short Form}

To investigate the developmental trajectories in infant temperament, mothers completed the Infant Behavior Questionnaire-Revised Very Short Form (IBQ-R VSF; Putnam et al., 2014), consisting of 37 items in total. For each question, mothers were asked to rate how often their infant had shown a specific behavior in a given situation during the past week by giving a score between 1 ("Never") and 7 ("Always"). Additionally, an item could be scored as "NA-Does not apply" should the situation described in the question not have occurred over the previous 7 days. The IBQ-R VSF can be separated into three broad scales: Surgency (13 items; e.g., "When tossed around playfully, how often did the baby laugh?"), Negative Affect (12 items; e.g., "At the end of an exciting day, how often did your baby become tearful?"), and Orienting/Regulatory Capacity (12 items; e.g., "How often during the last week did the baby enjoy being read to?") (Putnam et al., 2014). The IBQ-R VSF was scored according to Putnam et al. (2014). Average scores were calculated for each scale. Items rated as "NA-Does not apply" were excluded from analysis.

TABLE 1 Information on questionnaire completion rates and mean scores across the study's four postnatal stages for 63 participants

\begin{tabular}{lcccc} 
& 2 Weeks & 4 Months & 6 Months & 9 Months \\
\hline $\begin{array}{l}\text { Mean infant age (SD) at questionnaire } \\
\text { completion in days }\end{array}$ & $15.4(7.1)$ & $126.2(8.7)$ & $190.5(10.6)$ & $283.8(14.9)$ \\
\hline $\begin{array}{l}\text { Number of BDI-Ils completed } \\
\text { Mean scores for maternal BDI-II (SD) }\end{array}$ & 58 & 58 & 57 & 56 \\
\hline Number of IBQ-R VSFs complete & 59 & $8.81(7.55)$ & $7.83(8.09)$ & $7.05(7.82)$ \\
\hline $\begin{array}{l}\text { Mean scores (SD) for infant negative } \\
\text { affect (IBQ-R VSF) }\end{array}$ & $3.38(1.23)$ & $3.67(0.86)$ & $3.76(0.99)$ & $4.08(0.88)$ \\
\hline
\end{tabular}

Abbreviations: BDI-II, Beck Depression Inventory Second Edition; IBQ-R VSF, Infant Behavior Questionnaire-Revised Very Short Form; SD, standard deviation. 
Only scores on the Negative Affect scale were investigated in the present study, as the predicted association with maternal DS was specific to this scale.

Putnam et al. (2014) reported reliability scores for IBQ-R VSF scales derived from six previous studies using the full IBQ-R. The average Cronbach's alpha was .75 or above for the three broad IBQ-R VSF scales, and alpha specifically for the NA scale averaged .78, ranging from .72 to .88 across studies. Furthermore, two separate studies involving direct administration of the IBQ-R VSF found alpha values of .79 and .81 for NA (Putnam et al., 2014). In the current study, Cronbach's alpha for the NA scale ranged from .50 to .81 across ages, with a mean alpha of .67 (see Table S2).

The IBQ-R and IBQ-R VSF were developed and validated for infants aged 3-12 months (Putnam et al., 2014). However, previous research has indicated that these scales are suitable for younger infants as well, including newborns (Putnam et al., 2014; Worobey \& Blajda, 1989). In the current sample, Cronbach's alpha for NA at 2 weeks was .81, further demonstrating the suitability of the scale for very young infants.

\subsection{2 | The Beck Depression Inventory Second Edition}

To assess mothers' level of DS over the course of their infant's first year, we asked them to complete the Beck Depression Inventory Second Edition (BDI-II; Beck et al., 1996). The Edinburgh Postnatal Depression Scale (EPDS; Cox, Holden, \& Sagovsky, 1987) was also completed by the mothers when their infants were 9 months old as a complementary measure (see Supporting Information, Appendix 2, for details and results). The BDI-II is a standardized questionnaire containing 21 items. Each question asks to what extent a certain state does or does not apply to the respondent (e.g., Sadness; response options: 0-I do not feel sad, 1-I feel sad much of the time, 2-I am sad all the time, 3-I am so sad or unhappy that I cannot stand it). A higher score indicates a higher level of DS. A recent review of 118 studies established that the BDI-II had high internal consistency, with Cronbach's alpha ranging from .83 to .96 across both clinical and nonclinical samples (Wang \& Gorenstein, 2013). In the current study, Cronbach's alpha for BDI-II scores at the four ages ranged from .87 to .94 (see Table S2), with a mean alpha of .91. BDI-II questionnaires are scored by adding up the ratings for all items, with the first option always scoring zero and the last option always scoring three. The sum of scores indicates four different levels of depression. A score between 0 and 13 is classified as "minimal depression"; a score between 14 and 19 is classified as "mild depression"; a score between 20 and 28 is classified as "moderate depression"; and a score between 29 and 63 is classified as "severe depression" (Beck et al., 1996). Across the four ages, the majority of mothers scored in the minimally depressed category $(M=8.2, S D=7.4)$, with $82 \%$ of all scores falling in this range. The remaining scores were distributed as follows: $9.4 \%$ of scores indicated mild depression, $6.9 \%$ of scores indicated moderate depression, and $1.7 \%$ of scores indicated severe depression (see Table 2 for maternal BDI-II scores, at each depression level, across ages).

TAB LE 2 Breakdown of average BDI-II (Beck Depression Inventory Second Edition) scores at each maternal depression level and stage of the study for 63 participants

\begin{tabular}{|c|c|c|c|c|c|c|c|c|}
\hline & \multicolumn{2}{|l|}{2 Weeks } & \multicolumn{2}{|l|}{4 Months } & \multicolumn{2}{|l|}{6 Months } & \multicolumn{2}{|l|}{9 Months } \\
\hline & Average score & $N$ & Average score & $N$ & Average score & $N$ & Average score & $N$ \\
\hline Minimal (0-13) & 6.85 & 48 & 5.7 & 46 & 4.52 & 46 & 4.48 & 48 \\
\hline Mild (14-19) & 15.6 & 6 & 15.5 & 6 & 17.17 & 6 & 17 & 4 \\
\hline Moderate (20-28) & 22.33 & 3 & 23.8 & 5 & 24.25 & 4 & 24.67 & 3 \\
\hline Severe (29-63) & 29 & 1 & 37 & 1 & 38 & 1 & 38 & 1 \\
\hline
\end{tabular}




\subsection{3 | Parenting questionnaire}

Following the last lab visit, when infants were 9-10 months old, mothers were asked to complete a parenting questionnaire. It consisted of 7 items on parental feelings and 12 items on general attitudes to parenting. These were brief instruments based on scales developed by Budd et al. (2015) and by Deater-Deckard (2000), but also including some newly created items that were age appropriate for infants (see Supporting Information, Appendix S1, for further details). All questions were rated using a 5-point Likert scale with some items being reverse scored. Higher scores on the Parental Feelings scale indicated more positive feelings toward the child and satisfaction with the relationship with the child. Higher scores on the Parenting Attitudes scale indicated a less strict and punitive attitude to childrearing, while lower scores indicated a preference for harsher parenting. It should be noted that, in contrast to the IBQ-R VSF and the BDI-II, these scales were exploratory and have not been fully validated in their current form. Cronbach's alpha was .66 for the Parental Feelings scale and .53 for the Parenting Attitudes scale.

\section{3 | SEM analyses}

The data set was analyzed using path analysis within a structural equation modeling (SEM) framework. Five models were tested, each with different associations between maternal DS and infant NA at the four ages (see Introduction and Figures 1-5). Goodness-of-fit of each model was evaluated against the thresholds recommended by Hooper, Coughlan, and Mullen (2008) and Morin, Marsh, and Nagengast (2013). The fit was considered excellent if the comparative fit index (CFI) and the Tucker Lewis index $(T L I)>.95$, and the root mean square error of approximation (RMSEA) < .07. In addition, for adequate model fit, the Chi-square $\left(\chi^{2}\right)$ test was expected to be nonsignificant $(p>.05)$. For direct model comparisons, we used differences in Akaike Information Criterion (AIC) and the difference in Bayesian Information Criterion (BIC) values to assess whether there was weak evidence (0-2), positive evidence (2-6), strong evidence (6-10), or very strong evidence (10+) that one model fit the data better than another, in accordance with the guidelines provided by Wang and Wang (2012, p. 22). We also compared model fit using Satorra-Bentler scaled chi-square difference tests of nested models (Satorra \& Bentler, 2010). All analyses were performed using Mplus version 8 (Muthén \& Muthén, 2018). Full Information Maximum Likelihood estimation was used to account for missing data, and Robust Maximum Likelihood (MLR) was used to account for non-normality. The Mplus code for all five models can be found in the Supporting Information, Appendix S3, and on OSF: https://osf.io/mg68h/. A two-tailed alpha level of $p<.05$ was used to assess the significance of the paths in the best-fitting model.

\section{3 | RESULTS}

\section{1 | Descriptive statistics}

Means and standard deviations for maternal DS and infant NA at each stage are presented in Table 1. Table 2 provides a more detailed overview of BDI-II average scores grouped by four different levels of depression (minimal, mild, moderate, and severe).

\section{Zero-order correlations}

Table 3 presents the zero-order correlations between maternal BDI-II score and infant NA at each age. Across the study, maternal depression level and infant NA were moderately to highly stable (BDI-II $r$-value range: .48-.82, all 
TAB LE 3 Zero-order correlations between maternal Beck Depression Inventory Second Edition (BDI-II) score and infant negative affect (NA) scale score on the Infant Behavior Questionnaire-Revised Very Short Form (IBQ-R VSF) at each assessment stage

\begin{tabular}{|c|c|c|c|c|c|c|c|c|c|}
\hline & & \multicolumn{2}{|l|}{2 Weeks } & \multicolumn{2}{|c|}{4 Months } & \multicolumn{2}{|c|}{6 Months } & \multicolumn{2}{|c|}{9 Months } \\
\hline & & BDI-II & NA & BDI-II & NA & BDI-II & NA & BDI-II & NA \\
\hline \multirow[t]{2}{*}{2 Weeks } & BDI-II & 1 & & & & & & & \\
\hline & NA & $.317^{* *}$ & 1 & & & & & & \\
\hline \multirow[t]{2}{*}{4 Months } & BDI-II & $.528^{* * *}$ & .229 & 1 & & & & & \\
\hline & NA & $.382^{* *}$ & $.471^{* * *}$ & .165 & 1 & & & & \\
\hline \multirow[t]{2}{*}{6 Months } & BDI-II & $.478^{* * *}$ & .129 & $.754^{* * *}$ & .042 & 1 & & & \\
\hline & NA & $.357^{* *}$ & $.414^{* * *}$ & $.483^{* * *}$ & $.593^{* * *}$ & .221 & 1 & & \\
\hline \multirow[t]{2}{*}{9 Months } & BDI-II & $.630^{* * *}$ & $.311^{* *}$ & $.678^{* * *}$ & $.249^{*}$ & $.818^{* * *}$ & $.255^{*}$ & 1 & \\
\hline & NA & $.265^{*}$ & $.351^{* *}$ & $.480^{* * *}$ & $.506^{* * *}$ & $.295^{* *}$ & $.608^{* * *}$ & $.428^{* * *}$ & 1 \\
\hline
\end{tabular}

Note: Significant cross-measure correlations are in bold.

${ }^{* * *} p<.001 ;{ }^{* *} p<.01 ;{ }^{*} p<.05$, two-tailed $p$ values.

ps < .001; IBQ-R VSF r-value range: .35-.61, all ps < .01). In terms of the longitudinal association between maternal DS and infant NA, as can be seen in Table 3 , a higher BDI-II score at 2 weeks was associated with higher infant NA at all ages, with a peak in predictive association at 4 months and a slight decline in association toward the end of the first year, at 9 months. This lends support to the idea that maternal depression level around the time of birth is of particular importance to the development of infant NA, consistent with Model 1 (Sensitive Period Effects of Maternal DS). However, it is also clear from Table 3 that maternal BDI-II score at later ages too was significantly associated with infant NA at subsequent age points. Most noticeable are the associations between BDI-II score at 4 months and infant NA at 6 and 9 months; both correlations are $r>.45$. This is the case despite nonsignificant associations between maternal BDI-II score and infant NA within age at 4 months ( $r=.165)$ and 6 months $(r=.221)$. Simple correlation analyses are therefore suggestive of delayed effects of maternal DS beyond the immediate postnatal period, consistent with Model 2 (Delayed Effects of Maternal DS).

The zero-order correlations also suggested some evidence for small-to-moderate effects of infant NA on maternal DS, consistent with Model 3 (Delayed Effects of Infant NA). These were confined to predictive effects of earlier infant NA specifically on BDI-II score at 9 months (there were no significant longitudinal associations between infant NA at 2 weeks and 4 months and BDI-II scores at 4 or 6 months). On the whole, associations in this direction were smaller and less consistent. Nevertheless, as longitudinal effects were seen in both directions (infant-driven as well as maternally-driven), the Bi-directional model (Model 5) might be expected to show a good fit.

\section{2 | Path analysis results}

Of the five models, only Model 2 (Delayed Effects of Maternal DS) had an adequate fit to the data $\left(\chi^{2}(11)=15.66\right.$, $p=.15 ; \mathrm{RMSEA}=.08 ; \mathrm{CFI}=.98 ; \mathrm{TLI}=.95 ; \mathrm{BIC}=2083.60 ; \mathrm{AIC}=2012.87$ ), confirming delayed effects of maternal DS on infant NA across the first year of life. The model fit for the other models was inadequate (see Table 4). We performed Satorra-Bentler scaled chi-square difference tests of nested models with non-normal data (Satorra \& Bentler, 2010) for all of the appropriate model comparisons (see Supporting Information, Table S3). These tests showed that Model 2 achieved a better model fit than all of the other models except Model 5, where the chisquare difference was not significant. However, as Model 5 did not have superior fit and Model 2 is the more parsimonious model, Model 2 is preferred. 
TAB LE 4 Model fit indices for the five models (see Figures 1-5)

\begin{tabular}{|lllllllll} 
& $\chi 2$ & $d f$ & $p$ & CFI & TLI & RMSEA & BIC & AIC \\
\hline $\begin{array}{c}\text { Model 1: Sensitive period } \\
\text { effects of maternal } \\
\text { depressive symptoms }\end{array}$ & 35.096 & 13 & .0008 & .903 & .799 & .164 & $2,091.286$ & $2,024.849$ \\
$\begin{array}{c}\text { Model 2: Delayed effects } \\
\text { of maternal depressive }\end{array}$ & 15.659 & 11 & .1543 & .980 & .950 & .082 & $2,083.596$ & $2,012.872$ \\
$\begin{array}{c}\text { symptoms } \\
\text { Model 3: Delayed effects }\end{array}$ & 47.223 & 11 & $<.0001$ & .842 & .611 & .229 & $2,103.729$ & $2,033.005$ \\
\hline $\begin{array}{c}\text { of infant negative affect } \\
\text { Model 4: Stability model }\end{array}$ & 44.595 & 16 & .0002 & .875 & .789 & .168 & $2,086.676$ & $2,026.668$ \\
\hline $\begin{array}{c}\text { Model 5: Bi-directional } \\
\text { model }\end{array}$ & 14.865 & 6 & .0213 & .961 & .826 & .153 & $2,100.983$ & $2,019.543$ \\
\hline
\end{tabular}

Note: The best-fitting model is indicated in bold.

Abbreviations: AIC: Akaike information criterion; BIC: Bayesian information criterion; CFI: comparative fit index; $d f$ : degrees of freedom; $p$ : $p$ value for Chi-square statistic; RMSEA: root mean square error of approximation; TLI: Tucker Lewis index; $\chi^{2}$ : Chi-square statistic.

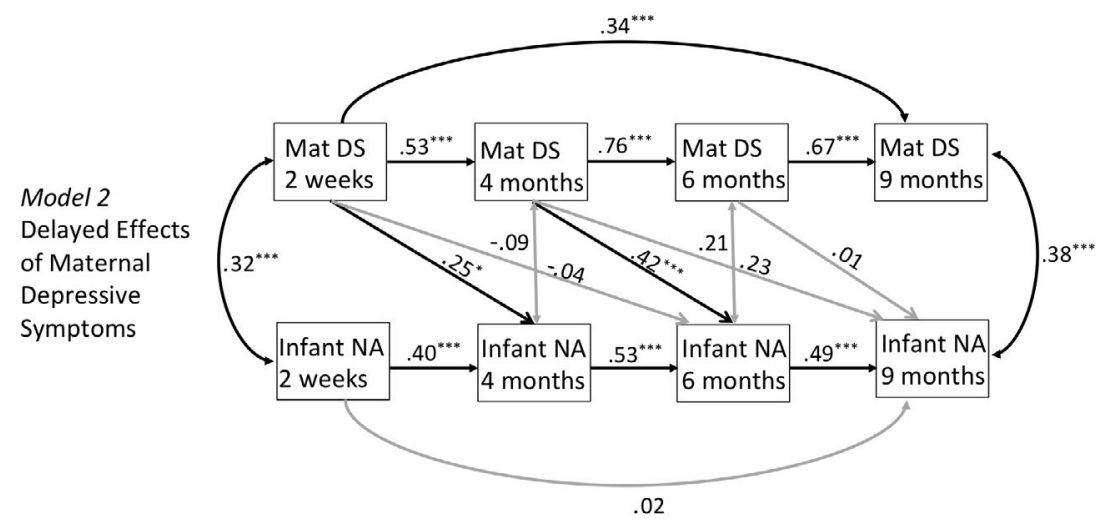

FIGURE 6 Best-fitting model of the relationship between maternal depressive symptoms (Mat DS) and infant negative affect (Infant NA). Standardized estimates are reported. Grey lines represent non-significant paths. ${ }^{* * *} p<.001,{ }^{* *} p<.01,{ }^{*} p<.05$, two-tailed

Unstandardized estimates from Model 2 can be found in Table S4, and 95\% confidence intervals around the path estimates in Model 2 using bootstrapping can be found in Table S6 (both in the Supporting Information). Table S5 reports the model fit indices for the five models, excluding one mother who scored consistently high on the BDI-II.

The best-fitting model (Model 2, see Figure 6) showed that maternal DS at 2 weeks was significantly predictive of infant NA at 4 months $(p=.032$ ). A new effect emerged at 4 months (which could not be accounted for by maternal DS at 2 weeks), with maternal depression level at this age significantly predicting infant NA at 6 months $(p<.001)$. No other predictive paths between maternal DS and infant NA reached significance. Maternal DS and infant NA were significantly associated within-age at 2 weeks and 9 months (both ps $<.001$ ), but not at 4 and 6 months. 


\section{3 | Power analysis (Monte Carlo simulation)}

Given the relatively small sample size, we carried out a Monte Carlo simulation (Muthén \& Muthén, 2002) to establish that we had adequate power to detect the effects reported for our best-fitting Model 2. In this analysis, we estimated models from 1,000 randomly generated samples. To be conservative, we input the smallest effects we detected in our final model with our observed sample size of $N=63$ (smallest autoregressive path estimate (same variable) $=.40$, and smallest regression path estimate (different variables) $=.25$ ). Model 2 achieved an average power of $74 \%$. Furthermore, we were able to detect effects with a power above $84 \%$ in two thirds of the paths, which is higher than the suggested power of $80 \%$ in behavioral sciences research (Cohen, 1988). The Monte Carlo simulation therefore indicated that for the majority of paths, we were adequately powered to detect the results reported for Model 2. In contrast, the more complex Bi-directional model (Model 5) achieved average power of only $65 \%$, suggesting that Model 5 was somewhat underpowered to detect effects.

\subsection{Associations between maternal DS, infant NA, and parenting measures}

To explore overall associations with parenting variables, correlations were calculated between scores from the two scales of the Parenting Questionnaire (for descriptive statistics, see Table 5) and maternal BDI-II scores and infant NA, averaged across ages. The analysis was done with BDI-II and NA scores averaged across age in order to reduce the number of bivariate analyses and because parenting variables were only available at 9 months. The correlation matrix can be seen in Table 6. This analysis was also run excluding one mum who scored consistently high on the BDI-II (see Supporting Information, Table S7).

As can be seen from Table 6, Parental Feelings score was significantly negatively correlated with the averaged BDI-II score across the study ages ( $r=-.395, p=.003$ ), that is, a lower level of maternal DS was associated with more positive feelings toward the child and satisfaction with the mother-child relationship. The effect was attenuated and only marginally significant $(r=-.260, p=.06)$ when the mother who scored consistently high on the BDI-II was excluded from the analysis (Table S7). Parental Feelings score was not significantly associated with overall infant NA. Furthermore, the other parenting scale, Parenting Attitudes, was not significantly correlated with either maternal DS or infant NA. However, it should be noted that the Parenting Attitudes scale had relatively low internal consistency $(\alpha=.53)$, suggesting that further work is needed to optimize this scale.

TABLE 5 Mean scores for the two scales of the Parenting Questionnaire

\begin{tabular}{|lll} 
& Number of items & Mean score (SD) \\
\hline Parental Feelings & 7 & $29.63(4.29)$ \\
\hline Parenting Attitudes & 12 & $47.16(4.67)$ \\
\hline
\end{tabular}

TAB LE 6 Zero-order correlations between the averaged maternal BDI-II (Beck Depression Inventory Second Edition) scores and infant negative affect (NA) scores (assessed by the Infant Behavior Questionnaire-Revised Very Short Form) and the two scales of the Parenting Questionnaire (assessed only at 9-10 months)

\begin{tabular}{lllll} 
& BDI-Il across stages & NA across stages & Parental Feelings & Parenting ttitudes \\
\hline BDI-II across stages & 1 & & & \\
NA across stages & $.399^{* *}$ & 1 & 1 & \\
\hline Parental Feelings & $-.395^{* *}$ & -.184 & .148 & 1 \\
\hline Parenting Attitudes & -.095 & .162 & \\
\hline
\end{tabular}

Note: Significant cross-measure correlations are in bold.

${ }^{* *} p<.01$, two-tailed $p$ values. 


\section{4 | DISCUSSION}

This study aimed to investigate the relationship between DS that mothers from a general population sample experience after childbirth, and infant NA across the first year of life. We tested five models to evaluate the direction of such associations. Based on previous evidence that showed maternally-driven effects (e.g., Pesonen et al., 2008; Shapiro et al., 2018; Sugawara et al., 1999), we expected maternal DS to predict infant NA across the first year of life. More specifically, we tested whether maternal DS soon after childbirth would either have a lasting effect on NA across the first year of life (Sensitive Period Effects of Maternal DS, Model 1), or whether effects would be cascading, with new effects of maternal DS emerging a few months after a change in maternal mood (Delayed Effects of Maternal DS, Model 2). Based on alternative evidence for infant-driven effects (Cutrona \& Troutman, 1986; Murray et al., 1996), we also tested a model with infant NA being the primary driver of later maternal DS (Delayed Effects of Infant NA, Model 3). Finally, a Stability Model (Model 4) was included as a control, and a more complex Bi-directional Model (Model 5) was also tested. The Bi-directional Model included all the paths tested in Models 2 and 3 , and therefore involved longitudinal associations going both from early maternal DS to later infant NA and from early infant NA to later maternal DS.

Zero-order correlations primarily indicated significant associations between maternal DS at birth and infant NA up to 9 months of age, and also between maternal DS at later ages and infant NA at subsequent age points. There were also some significant associations between early infant NA and later maternal DS, although these were weaker and less consistent. Subsequent path analyses allowed us to test which of the five models fit the data best, and thereby provided an estimation of the directionality and time course of the associations seen in the zero-order correlations, while at the same time controlling for within-age associations between the two primary variables. It was found that Model 2 (Delayed Effects of Maternal DS) fit the data best. The path coefficients from the best-fitting model indicated that, not only do maternal DS at 2 weeks postpartum significantly predict infant NA up to 4 months of age, but also that a new effect of maternal DS emerges at 4 months, predicting infant NA at 6 months.

Our results are in line with those that indicate the important impact of maternal DS across a child's early development (Brennan et al., 2000; Campbell, Matestic, Stauffenberg, Mohan, \& Kirchner, 2007; Field, 1992; Tronick, 1989). We found that changes in maternal depression level across the first year were associated with concurrent and later infant NA. The existence of a strong association between maternal DS and infant NA already at 2 weeks after birth suggests that maternal mood and infant mood are closely linked from very early on in the infant's life. However, other effects were delayed, such that a change in maternal mood was associated with a change in infant NA a few months later. Delayed associations between maternal DS and child temperament have been found in the past (Pesonen et al., 2008; Shapiro et al., 2018; Sugawara et al., 1999), however, typically these studies only assessed maternal DS and child temperament at one or two points in time, often years apart. For example, Pesonen et al. (2008) reported level of maternal stress (which has some, but not complete, overlap with maternal DS) and child NA at two time points only. In that study, maternal stress at 6 months postpartum predicted the level of child NA at 5 years of age. Our study, examining both maternal DS and infant NA using the same well-validated measures across the very earliest part of infant development, allowed us to shed further light on the time course of the complex interactions between these factors from birth onward, revealing not only effects of maternal mood on infant temperament at birth, but also cascading effects of maternal DS on infant NA across the first 9 months of life. Our study also confirmed the directionality of the primary effect found by several previous studies (Pesonen et al., 2008; Shapiro et al., 2018; Sugawara et al., 1999), that is, maternal DS predicting infant NA.

It should be noted that we only found delayed effects of maternal DS between 2 weeks and 4 months and between 4 and 6 months. There were no delayed effects of maternal DS at 4 and 6 months on infant NA at 9 months. We can only speculate as to the reasons that cascade effects only appeared during the first 6 months. One possibility is that no new cascade effects of maternal DS appear after 6 months, once the earlier effects are accounted for in the model. Alternatively, we may not have had enough power to detect a more subtle effect later in infancy. 
It is worth noting, however, that the association between maternal DS and infant NA within-age at 9 months is significant (see Figure 6), suggesting that there continues to be an association between maternal DS and infant NA and that longitudinal studies with a longer follow-up period may reveal further cascades. Another possibility is that, as infants experience an expanded social environment during the second half of the first year of life, a time where many infants start attending nursery or other daycare in the U.K., the influence of maternal DS becomes less pronounced. Further research is needed to establish the potentially changing association between maternal DS and infant temperament across such transitions.

In the current study, a model with longitudinal paths going only from infant NA to maternal DS (Model 3) did not fit the data well, suggesting that, contrary to some prior evidence (Cutrona \& Troutman, 1986; Murray et al., 1996), infant expression of NA in the early days did not elicit DS in the mother later in the first year. A few caveats must be considered, however, before ruling out such effects. The studies by Cutrona and Troutman (1986) and Murray et al. (1996) involved mothers and infants in the early postnatal period (within the first 3 months). Consistent with this, we did see a highly significant zero-order correlation between maternal DS and infant NA at 2 weeks, and the directionality of this effect cannot be established within the same age;so it is possible that infant NA predicted maternal DS (rather than the other way round) in the early postnatal weeks. Furthermore, the study by Murray et al. (1996) involved mothers at high risk of developing depression, and it is conceivable that such mothers may be more vulnerable to difficult infant temperament. Finally, we did see some zero-order longitudinal correlations between infant NA and maternal DS, albeit less consistently, and it is possible that with a larger sample size, we would have been able to detect these effects in our model.

Relatedly, Model 5, which involved both maternally-driven and infant-driven effects, did also not have an adequate fit, suggesting that adding the longitudinal paths from infant NA to maternal DS did not improve the model. However, with a sample size of 63 we had limited power to test such a complex model, and therefore, to detect potentially more subtle bi-directional effects over time (cf., the Monte Carlo simulation results on p. 12). We therefore see the current study as a first step toward larger studies that follow up on and replicate cascading effects across the first year and further explore the directionality of these effects. It is entirely possible that more subtle infant-driven effects could be detected in such studies. In fact, consistent with this, and consistent with our own zero-order correlations, Pesonen et al. (2008) did find some infant-driven effects (across a 5-year period), but these effects were substantially weaker than maternally-driven effects and were specific to NA subscales rather than the overall NA construct. Further research is therefore needed to establish when infant-driven effects on maternal DS come into play and the magnitude of these effects.

Our results support the findings from two recent studies which, like the current study, looked at trajectories of maternal DS in nonclinical populations. Cents and colleagues (2013) showed that even when symptom severity is low, exposure to maternal DS predicts child behavioral problems at 3 years of age. Likewise, Giallo et al. (2015) found that children exposed to persistent DS even at low levels ("subclinical DS" group) had similar levels of emotional and behavioral difficulties to children of mothers who reported "increasing and persistently high DS" from pregnancy to 4 years postpartum. Children in the subclinical DS group were also significantly more likely to experience difficulties than children of mothers reporting no or minimal symptoms (Giallo et al., 2015). Altogether, the findings from these studies highlight the importance of focusing not only on clinically depressed mothers, but also on that considerable proportion of women who experience subclinical postnatal DS that are likely to remain undetected by practitioners. These subclinical symptoms might in fact have a similar adverse long-term impact on a child's emotional and behavioral development as the more severe symptoms associated with clinical maternal depression. The current study specifically contributes to this conclusion by showing that maternal (primarily subclinical) DS already have cascading effects early on in a child's life. It is a question for future research how the cascading effects reported here may develop beyond the first year. Measures of long-term outcomes, as well as direct measures of mother-infant interaction, would also be of particular relevance in order to further support the present findings and their implications for early development. 
It is important to consider that the current study primarily addressed the longitudinal associations between maternal DS and infant NA, and which model describes these effects best. Our data do not provide information on the mechanisms underlying the associations we found. A large literature exists, both theoretical (e.g., Cummings \& Davies, 1994; Goodman \& Gotlib, 1999; Tronick, 1989) and empirical (e.g., Beebe et al., 2008; Feldman et al., 2009; Granat et al., 2017), which describes these mechanisms, in particular the potential influence of parenting factors such as lowered maternal sensitivity and dysfunctional interaction patterns on producing negative cognitive and affective outcomes in children. As we did not collect longitudinal data on mother-infant interaction, we cannot assess whether such mechanisms were at play. An important avenue for future research is therefore the repeated assessment of maternal DS and infant NA alongside direct observation of mother-infant interaction. Such research would allow identification of the key factors that underlie the cascading effects of maternal DS on infant NA that we observed in the current study. These factors could be emotional aspects of the interaction (e.g., comfort, maternal sensitivity) or more cognitive aspects (e.g., eye contact, gaze and action synchrony, cognitive stimulation, and engagement) or both.

Although longitudinal mother-infant interaction measures were not available, we did collect some basic parenting measures at 9 months of age, and found that mothers with a high level of DS also tended to perceive the relationship with their baby more negatively and find it harder to cope with parenting. This is consistent with previous research (e.g., Cutrona \& Troutman, 1986; Feldman et al., 2009). Interestingly, there was no overall association between the mother's perception of the relationship with her child and infant NA, perhaps suggesting that parental feelings about the relationship may not be the main factor mediating the link between maternal DS and infant NA. However, to fully establish the relationships between maternal DS, parenting, and infant NA, longitudinal data on all three variables are needed.

The current study had a number of limitations. We did not include prenatal measures of maternal depression. Another question that remains open is therefore to what extent prenatal DS influenced our findings. We cannot rule out the possibility that the association between maternal DS and infant NA at birth is instead a biologically mediated carryover effect from prenatal DS. A considerable amount of evidence exists demonstrating that prenatal depression impacts on the fetus and subsequent infant development, including the development of NA (see, Field, Diego, \& Hernandez-Reif, 2006, for a review). However, the additional effect emerging around 4 months of age in our study cannot be explained by the same biological mechanism. Ideally, future research should include measures of both prenatal and postnatal maternal DS to investigate the relationship between maternal mood and the development of infant temperament more comprehensively.

Another limitation that is worth considering is the fact that only a single informant provided data in the current study (i.e., the mother). Although this is a potential concern, researchers in the fields of maternal depression and infant temperament have highlighted that the accuracy and importance of maternal ratings should not be overlooked (Brennan et al., 2000; Goodman et al., 2011; Richters, 1992; Rothbart, 2011). Furthermore, the pattern of associations seen in the current data set do not support a general "negativity bias," whereby mothers with a higher level of DS simply rate their infants as having more NA overall. This is because maternal DS and infant NA were not always significantly associated within-age (see Table 3 and Figure 6), which would have been the case if the mother's mood directly reflected her ratings of her infant's NA when the ratings were made. In contrast, the longitudinal effects were robust, despite these ratings being made several months apart. Nonetheless, associations between any two variables are likely to be higher and to be affected by a number of intrapersonal factors when one person is reporting on those two variables rather than when the data come from different sources, and this is an important limitation of our study. Ratings from fathers (and other caregivers) will be important to establish whether effects are robust across raters. Furthermore, to bolster the present findings, future studies should include direct observations of maternal and infant behavior longitudinally. This would not only allow confirmation of the current results across different temperament assessment methods, but also a better understanding of the fine-grained interpersonal mechanisms involved, such as social responsiveness and mother-infant communicative synchrony (e.g., Beebe et al., 2008; Feldman et al., 2009; Field, 1984). 
Relatedly, as our data set did not include repeated assessments of parenting attitudes and behavior, maternal self-esteem, social support, and other personality and social factors, we cannot estimate the potential mediation of our results by such variables. For example, it is possible that low maternal sensitivity to the infant's communicative signals is one of the underlying factors in the cascading associations we observe (Feldman et al., 2009), but that with good social support and buffering effects from other caregivers (Cutrona \& Troutman, 1986) such effects are attenuated. These are important factors to consider, and their incorporation in future research will allow a fuller picture of the dynamic interplay between maternal DS and infant NA across development.

In the present study, we have not considered the potential effect of paternal DS (and the potential interaction between maternal and paternal DS) on early infant temperament, but this is an important area for future research and one that has been somewhat neglected in the field until recently (for review, see Gentile \& Fusco, 2017; Sweeney \& MacBeth, 2016). We also cannot assume that effects would be the same in a clinically depressed population, which is an important question from an intervention perspective. Finally, our research was carried out in a group of mothers from relatively affluent backgrounds, and further research is needed to establish whether effects are the same in mothers at higher socioeconomic risk.

These limitations aside, the current study provides novel insights into the significant association between maternal DS and infant temperament using consistently collected longitudinal data in a typical population. The cascading effects we found across four time points during the first year of life have not been demonstrated in previous studies. This type of information is of relevance for developing support and intervention programs that could be helpful for the general population of parents and parents-to-be rather than specifically focusing only on mothers who have been diagnosed by a professional as being depressed or those at risk of depression. The finding that effects were cascading means that continuous support across early infancy is likely to be important, not just during the first postnatal weeks. Further research adopting a longitudinal approach, involving both questionnaire responses and direct observations in a larger sample, may help further establish the time course and mechanisms underlying these cascading effects, both in and beyond the first year of life.

\section{ACKNOWLEDGMENTS}

The research was supported by a Grand Challenges Explorations grant from the Bill \& Melinda Gates Foundation to S.R. and K.H. (OPP1119448). We would like to thank all the families who took part in the study and who thereby made this research possible.

\section{CONFLICT OF INTEREST}

The authors declare no conflicts of interest.

\section{DATA AVAILABILITY STATEMENT}

The data and analysis scripts that support the findings of this study are openly available on OSF, at https://osf.io/ mg68h/ [https://doi.org/10.17605/OSF.IO/MG68H].

\section{ORCID}

Silvia Rigato (iD https://orcid.org/0000-0002-3470-6752

Manuela Stets iD https://orcid.org/0000-0001-8078-7357

Arielle Bonneville-Roussy iD https://orcid.org/0000-0001-7909-8845

Karla Holmboe iD https://orcid.org/0000-0003-3157-6101

\section{REFERENCES}

Ainsworth, M. S., Blehar, M. C., Waters, E., \& Wall, S. (1978). Patterns of attachment: A psychological study of the strange situation. Oxford, UK: Erlbaum. 
Beck, A. T., Steer, R. A., \& Brown, G. K. (1996). Manual for the Beck Depression Inventory-II. San Antonio, TX: Psychological Corporation.

Beck, C. T. (1996). A meta-analysis of the relationship between postpartum depression and infant temperament. Nursing Research, 45(4), 225-230. https://doi.org/10.1097/00006199-199607000-00006

Beck, C. T. (1998). The effects of postpartum depression on child development: A meta-analysis. Archives of Psychiatric Nursing, 12, 12-20. https://doi.org/10.1016/s0883-9417(98)80004-6

Beebe, B., Badalamenti, A., Jaffe, J., Feldstein, S., Marquette, L., Helbraun, E., ... Ellman, L. (2008). Distressed mothers and their infants use a less efficient timing mechanism in creating expectancies of each other's looking patterns. Journal of Psycholinguistic Research, 37(5), 293-307. https://doi.org/10.1007/s10936-008-9078-y

Belsky, J., Putnam, S., Crnic, K., \& (1996). Coparenting, parenting, and early emotional development. In J. P. Hale \& P. A. Cowan (Eds.), Understanding how family-level dynamics affect children's development: Studies of two-parent families (pp. 45-55). San Francisco, CA: Jossey-Bass. https://doi.org/10.1002/cd.23219967405

Brennan, P. A., Hammen, C., Andersen, M. J., Bor, W., Najman, J. M., \& Williams, G. M. (2000). Chronicity, severity, and timing of maternal depressive symptoms: Relationships with child outcomes at age 5. Developmental Psychology, 36(6), 759-766 . https://doi.org/10.1037//0012-1649.36.6.759

Budd, K. S., Danko, C. M., Li, Y., \& Henriquez, S. (2015). Assessing childrearing attitudes: Validation of a shortened version of the parenting questionnaire. Parenting, 15(1), 39-54. https://doi.org/10.1080/15295192.2015.992737

Campbell, S. B., Cohn, J. F., \& Meyers, T. (1995). Depression in first-time mothers: Mother-infant interaction and depression chronicity. Developmental Psychology, 31, 349-357. https://doi.org/10.1037/0012-1649.31.3.349

Campbell, S. B., Matestic, P., von Stauffenberg, C., Mohan, R., \& Kirchner, T. (2007). Trajectories of maternal depressive symptoms, maternal sensitivity, and children's functioning at school entry. Developmental Psychology, 43(5), 12021215. https://doi.org/10.1037/0012-1649.43.5.1202

Campbell, S. B., Morgan-Lopez, A. A., Cox, M. J., McLoyd, V. C., \& NICHD-ECRN. (2009). A latent class analysis of maternal depressive symptoms over 12 years and offspring adjustment in adolescence. Journal of Abnormal Psychology, 118(3), 479-493. https://doi.org/10.1037/a0015923

Cents, R. A. M., Diamantopoulou, S., Hudziak, J. J., Jaddoe, V. W. V., Hofman, A., Verhulst, F. C., ... Tiemeier, H. (2013). Trajectories of maternal depressive symptoms predict child problem behaviour: The Generation R study. Psychological Medicine, 43(1), 13-25. https://doi.org/10.1017/S0033291712000657

Cohen, J. (1988). Statistical power analysis for the behavioral sciences. Abingdon, UK: Routledge.

Cox, J. L., Holden, J. M., \& Sagovsky, R. (1987). Detection of postnatal depression: Development of the 10-item Edinburgh postnatal depression scale. British Journal of Psychiatry, 150, 782-786. https://doi.org/10.1192/bjp.150.6.782

Cummings, E. M., \& Davies, P. T. (1994). Maternal depression and child development. Journal of Child Psychology and Psychiatry, 35(1), 73-122. https://doi.org/10.1111/j.1469-7610.1994.tb01133.x

Cutrona, C. E., \& Troutman, B. R. (1986). Social support, infant temperament, and parenting self-efficacy: A mediational model of postpartum depression. Child Development, 57, 1507-1518. https://doi.org/10.2307/1130428

Deater-Deckard, K. (2000). Parenting and child behavioral adjustment in early childhood: A quantitative genetic approach to studying family processes and child development. Child Development, 71, 468-484. https://doi. org/10.1111/1467-8624.00158

Feldman, R., Granat, A., Pariente, C., Kanety, H., Kuint, J., \& Gilboa-Schechtman, E. (2009). Maternal depression and anxiety across the postpartum year and infant social engagement, fear regulation, and stress reactivity. Journal of the American Academy of Child and Adolescent Psychiatry, 48(9), 919-927. https://doi.org/10.1097/CHI.0b013e3181 b21651

Field, T. (1984). Early interactions between infants and their postpartum depressed mothers. Infant Behavior and Development, 7, 517-522. https://doi.org/10.1016/S0163-6383(84)80010-7

Field, T. (1992). Infants of depressed mothers. Development and Psychopathology, 4(1), 49-66. https://doi.org/10.1017/ S0954579400005551

Field, T., Diego, M., \& Hernandez-Reif, M. (2006). Prenatal depression effects on the fetus and newborn: A review. Infant Behavior and Development, 29(3), 445-455. https://doi.org/10.1016/j.infbeh.2006.03.003

Field, T., Healy, B. T., Goldstein, S., \& Guthertz, M. (1990). Behavior-state matching and synchrony in mother-infant interactions of nondepressed versus depressed dyads. Developmental Psychology, 26(1), 7-14. . https://doi. org/10.1037/0012-1649.26.1.7

Gentile, S., \& Fusco, M. L. (2017). Untreated perinatal paternal depression: Effects on offspring. Psychiatry Research, 252, 325-332. https://doi.org/10.1016/j.psychres.2017.02.064

Giallo, R., Woolhouse, H., Gartland, D., Hiscock, H., \& Brown, S. (2015). The emotional-behavioural functioning of children exposed to maternal depressive symptoms across pregnancy and early childhood: A prospective Australian pregnancy cohort study. European Child \& Adolescent Psychiatry, 24(10), 1233-1244. https://doi.org/10.1007/ s00787-014-0672-2 
Goldsmith, H. H., Buss, A. H., Plomin, R., Rothbart, M. K., Thomas, A., Chess, S., \& McCall, R. B. (1987). Roundtable: What is temperament? Four approaches. Child Development, 58, 505-529.https://doi.org/10.2307/1130527.

Goodman, S. H., \& Gotlib, I. H. (1999). Risk for psychopathology in the children of depressed mothers: A developmental model for understanding mechanisms of transmission. Psychological Review, 106(3), 458-490. https://doi. org/10.1037/0033-295x.106.3.458

Goodman, S. H., Rouse, M. H., Connell, A. M., Broth, M. R., Hall, C. M., \& Heyward, D. (2011). Maternal depression and child psychopathology: A meta-analytic review. Clinical Child and Family Psychology Review, 14, 1-27. https://doi. org/10.1007/s10567-010-0080-1

Gordon, D., Burge, D., Hammen, C., Adrian, C., Jaenicke, C., \& Hiroto, D. (1989). Observations of interactions of depressed women with their children. The American Journal of Psychiatry, 146(1), 50-55. . https://doi.org/10.1176/ajp.146.1.50

Granat, A., Gadassi, R., Gilboa-Schechtman, E., \& Feldman, R. (2017). Maternal depression and anxiety, social synchrony, and infant regulation of negative and positive emotions. Emotion, 17(1), 11-27. https://doi.org/10.1037/emo0000204

Hooper, D., Coughlan, J., \& Mullen, M. R. (2008). Structural equation modelling: Guidelines for determining model fit. Electronic Journal of Business Research Methods, 6(1), 53-60.

Horwitz, S. M., Briggs-Gowan, M. J., Storfer-Isser, A., \& Carter, A. S. (2009). Persistence of maternal depressive symptoms throughout the early years of childhood. Journal of Women's Health, 18(5), 637-645. https://doi.org/10.1089/ jwh.2008.1229

Howard, L. M., Molyneaux, E., Dennis, C. L., Rochat, T., Stein, A., \& Milgrom, J. (2014). Non-psychotic mental disorders in the perinatal period. Lancet, 384(9956), 1775-1788. https://doi.org/10.1016/S0140-6736(14)61276-9

Kingston, D., McDonald, S., Austin, M. P., \& Tough, S. (2015). Association between prenatal and postnatal psychological distress and toddler cognitive development: A systematic review. PLoS ONE, 10(5), e0126929. https://doi. org/10.1371/journal.pone.0126929

Kingston, D., \& Tough, S. (2014). Prenatal and postnatal maternal mental health and school-age child development: A systematic review. Maternal and Child Health Journal, 18(7), 1728-1741. https://doi.org/10.1007/s10995-013-1418-3

Kingston, D., Tough, S., \& Whitfield, H. (2012). Prenatal and postpartum maternal psychological distress and infant development: A systematic review. Child Psychiatry \& Human Development, 43(5), 683-714. https://doi.org/10.1007/ s10578-012-0291-4

Kline, R. B. (2016). Principles and practice of structural equation modelling (4th ed.). New York, NY: The Guilford Press.

Kopp, C. B. (1989). Regulation of distress and negative emotions: A developmental view. Developmental Psychology, 25(3), 343-354. https://doi.org/10.1037/0012-1649.25.3.343

Lyons-Ruth, K., Connell, D. B., Grunebaum, H. U., \& Botein, S. (1990). Infants at social risk: Maternal depression and family support service as mediators of infant development and security of attachment. Child Development, 61(1), 85-98. https://doi.org/10.1111/j.1467-8624.1990.tb02762.x

McMahon, C., Barnett, B., Kowalenko, N., Tennant, C., \& Don, N. (2001). Postnatal depression, anxiety and unsettled infant behaviour. Australian \& New Zealand Journal of Psychiatry, 35(5), 581-588. https://doi.org/10.1080/0004867010 060505

Morin, A. J. S., Marsh, H. W., \& Nagengast, B. (2013). Exploratory structural equation modeling. In G. R. Hancock \& R. O. Mueller (Eds.), Structural equation modeling: A second course (2nd ed.). Greenwich, CT: IAP.

Murray, L., Halligan, S., \& Cooper, P. (2010). Effects of postnatal depression on mother-infant interactions and child development. In J. G. Bremner \& T. D. Wachs (Eds.), The Wiley-Blackwell handbook of infant development (2nd ed., Vol. 2). West Sussex, UK: Wiley-Blackwell. https://doi.org/10.1002/9781444327588.ch8

Murray, L., Stanley, C., Hooper, R., King, F., \& Fiori-Cowley, A. (1996). The role of infant factors in postnatal depression and mother-infant interactions. Developmental Medicine \& Child Neurology, 38(2), 109-119. https://doi. org/10.1111/j.1469-8749.1996.tb12082.x

Muthén, L. K., \& Muthén, B. O. (2002). How to use a Monte Carlo study to decide on sample size and determine power. Structural Equation Modeling: A Multidisciplinary Journal, 9(4), 599-620. https://doi.org/10.1207/S15328007S EM0904_8

Muthén, L. K., \& Muthén, B. O. (2018). Mplus version 8.0. Los Angeles, CA: Author.

Pawluski, J. L., Lonstein, J. S., \& Fleming, A. S. (2017). The neurobiology of postpartum anxiety and depression. Trends in Neurosciences, 40(2), 106-120. https://doi.org/10.1016/j.tins.2016.11.009

Perry, N. B., Dollar, J. M., Calkins, S. D., \& Bell, M. A. (2018). Developmental cascade and transactional associations among biological and behavioral indicators of temperament and maternal behavior. Child Development, 89(5), $1735-1751$. https://doi.org/10.1111/cdev.12842

Pesonen, A., Räikkönen, K., Heinonen, K., Komsi, N., Järvenpää, A., \& Strandberg, T. (2008). A transactional model of temperamental development: Evidence of a relationship between child temperament and maternal stress over five years. Social Development, 17, 326-340. https://doi.org/10.1111/j.1467-9507.2007.00427.x

Pickens, J., \& Field, T. (1993). Facial expressivity in infants of depressed mothers. Developmental Psychology, 29, 986-988. https://doi.org/10.1037/0012-1649.29.6.986 
Priest, S. R., Austin, M. P., Barnett, B. B., \& Buist, A. (2008). A psychosocial risk assessment model (PRAM) for use with pregnant and postpartum women in primary care settings. Archives of Women's Mental Health, 11(5-6), 307-317. . https://doi.org/10.1007/s00737-008-0028-3

Putnam, S. P., Helbig, A. L., Gartstein, M. A., Rothbart, M. K., \& Leerkes, E. (2014). Development and assessment of short and very short forms of the infant behavior questionnaire-revised. Journal of Personality Assessment, 96, 445-458. https://doi.org/10.1080/00223891.2013.841171

Richters, J. E. (1992). Depressed mothers as informants about their children: A critical review of the evidence for distortion. Psychological Bulletin, 112, 485-499. https://doi.org/10.1037/0033-2909.112.3.485

Rothbart, M. K. (2011). Becoming who we are: Temperament and personality in development. New York, NY: The Guilford Press.

Rothbart, M. K., \& Bates, J. E. (2006). Temperament. In N. Eisenberg, W. Damon, \& R. M. Lerner (Eds.), Handbook of child psychology: Vol. 3, Social, emotional, and personality development (6th ed., pp. 99-166). Hoboken, NJ: Wiley. https://doi. org/10.1002/9780470147658.chpsy0303

Satorra, A., \& Bentler, P. M. (2010). Ensuring positiveness of the scaled difference chi-square test statistic. Psychometrika, 75(2), 243-248. https://doi.org/10.1007/s11336-009-9135-y

Shapiro, A. F., Jolley, S. N., Hildebrandt, U., \& Spieker, S. J. (2018). The effects of early postpartum depression on infant temperament. Early Child Development and Care, 1-13. https://doi.org/10.1080/03004430.2018.1552947

Sugawara, M., Kitamura, T., Toda, M. A., \& Shima, S. (1999). Longitudinal relationship between maternal depression and infant temperament in a Japanese population. Journal of Clinical Psychology, 55(7), 869-880. https://doi.org/10.1002/ (SICI)1097-4679(199907)55:7<869::AID-JCLP8>3.0.CO;2-F

Sweeney, S., \& MacBeth, A. (2016). The effects of paternal depression on child and adolescent outcomes: A systematic review. Journal of Affective Disorders, 205, 44-59. https://doi.org/10.1016/j.jad.2016.05.073

Tronick, E. Z. (1989). Emotions and emotional communication in infants. American Psychologist, 44(2), 112-119. . https:// doi.org/10.1037//0003-066X.44.2.112

Wang, J., \& Wang, Q. (2012). Structural equation modeling: Applications using Mplus. West Sussex, UK: Wiley.

Wang, Y.-P., \& Gorenstein, C. (2013). Psychometric properties of the Beck Depression Inventory-II: A comprehensive review. Revista Brasileira de Psiquiatria, 35, 416-431. https://doi.org/10.1590/1516-4446-2012-1048

Weisman, O., Granat, A., Gilboa-Schechtman, E., Singer, M., Gordon, I., Azulay, H., ... Feldman, R. (2010). The experience of labor, maternal perception of the infant, and the mother's postpartum mood in a low-risk community cohort. Archives of Women's Mental Health, 13(6), 505-513. https://doi.org/10.1007/s00737-010-0169-z

Weissman, M. M., Wickramaratne, P., Gameroff, M. J., Warner, V., Pilowsky, D., Kohad, R. G., ... Talati, A. (2016). Offspring of depressed parents: 30 years later. American Journal of Psychiatry, 73(10), 1024-1032. https://doi.org/10.1176/appi. ajp. 2016.15101327

Whiffen, V. E., \& Gotlib, I. E. (1989). Infants of postpartum depressed mothers: Temperament and cognitive status. Journal of Abnormal Psychology, 98(3), 274-279. https://doi.org/10.1037//0021-843x.98.3.274

Woolhouse, H., Gartland, D., Mensah, F., \& Brown, S. J. (2015). Maternal depression from early pregnancy to 4 years postpartum in a prospective pregnancy cohort study: Implications for primary health care. BJOG: An International Journal of Obstetrics and Gynaecology, 122(3), 312-321. https://doi.org/10.1111/1471-0528.12837

Worobey, J., \& Blajda, V. M. (1989). Temperament ratings at 2 weeks, 2 months, and 1 year: Differential stablity of activity and emotionality. Developmental Psychology, 25(2), 257-263. https://doi.org/10.1037/0012-1649.25.2.257

\section{SUPPORTING INFORMATION}

Additional Supporting Information may be found online in the Supporting Information section.

Supplementary Material

How to cite this article: Rigato S, Stets M, Bonneville-Roussy A, Holmboe K. Impact of maternal depressive symptoms on the development of infant temperament: Cascading effects during the first year of life. Soc. Dev.. 2020;29:1115-1133. https://doi.org/10.1111/sode.12448 